\title{
Power aware MOM for telemetry-oriented applications using GPRS-enabled embedded devices - levee monitoring use case
}

\author{
Tomasz Szydlo *, Piotr Nawrocki ${ }^{\dagger}$ and Robert Brzoza-Woch ${ }^{\ddagger}$ and Krzysztof Zielinski ${ }^{\S}$ \\ *AGH University of Science and Technology \\ Faculty of Computer Science, Electronics and Telecommunications \\ Department of Computer Science \\ al. A. Mickiewicza 30, 30-059 Krakow, Poland \\ e-mail:tomasz.szydlo@agh.edu.pl \\ †e-mail:piter@agh.edu.pl \\ ‡e-mail:rabw@agh.edu.pl \\ $\S$ e-mail:kz@agh.edu.pl
}

\begin{abstract}
The paper proposes the concept of adaptive message aggregation for telemetry applications that use GPRS connectivity. The method optimizes the power consumed during data transmission, what is useful in the modern telemetry devices powered from renewable energy sources. The concept has been verified in the levee monitoring scenario.
\end{abstract}

\section{INTRODUCTION}

$\mathbf{T}$ HE PURPOSE of telemetry systems is to transparently convey measurement information from a remotely located sensor to receiving equipment for further processing and visualization. Development and miniaturization of electronic devices has allowed for the high penetration of telemetry solutions in the surrounding world in order to increase the quality of life. In typical telemetry solutions, remote stations are powered from external power sources and use industrial communication protocols such as Modbus to gather data from these devices to the central system. The communication to the remote location is achieved by the GPRS network as a low cost and easily accessible communication layer [1]. These protocols provide data by polling, so it requires the remote stations to be available all the time.

Currently, such devices are designed to be powered by energy harvesting thus they must be power efficient and they might temporarily go asleep to preserve power [2], [3]. Because of the differences between these types of devices, the legacy polling protocols might not be effective. We argue that the communication protocols should (1) leverage the power usage characteristic of GPRS technology, (2) handle the sleepy nodes and (3) provide high level addressing of nodes. We think that these requirements might be fulfilled by the message oriented communication. Sending messages across channels decreases the complexity of the end application, thereby allowing the developer of the application to focus on true application functionality instead of the intricate needs of communication protocols. Message-oriented middleware (MOM)[4] allows application modules to be distributed over heterogeneous platforms and reduces the complexity of developing applications that span multiple operating systems and network protocols. The middleware creates a distributed communications layer that insulates the application developer from the details of the various operating systems and network interfaces. Message-oriented middleware may provide reliable asynchronous communication mechanisms that might be used to carry i.e. measurement data or other remote communication messages.

In the paper we propose the concept of adaptive message aggregation method for MQTT-SN protocol that optimizes power used by GPRS wireless connection during data transmission. The preliminary research (presented later in the paper) showed that sending data using short IP packets consumes much more energy than using longer packets. Because of the fact that messages containing measurements are relatively small, we propose the concept of adaptive data aggregation prior to sending via GPRS.

The research presented in this paper is a part of ISMOP [5] research project which objectives span construction of an artificial levee, design of wireless sensors for levee instrumentation, development of a sensor communication infrastructure, and a software platform for execution management, data management [6] and decision support [7]. Scientific and industrial consortium in the ISMOP project conducts research on a comprehensive monitoring system enabling evaluation of current and forecasted state of flood levees. This paper focuses on issues related to the organization of data acquired from the sensors located in the levees in order to optimize the power consumed by GPRS modem during data transmission to the central system for later analysis.

The paper is organized as follows. Section II discusses the motivating scenario, where Section III presents the related work. Section IV presents the concept of power-aware adaptive message aggregation, which is then evaluated in the use case 


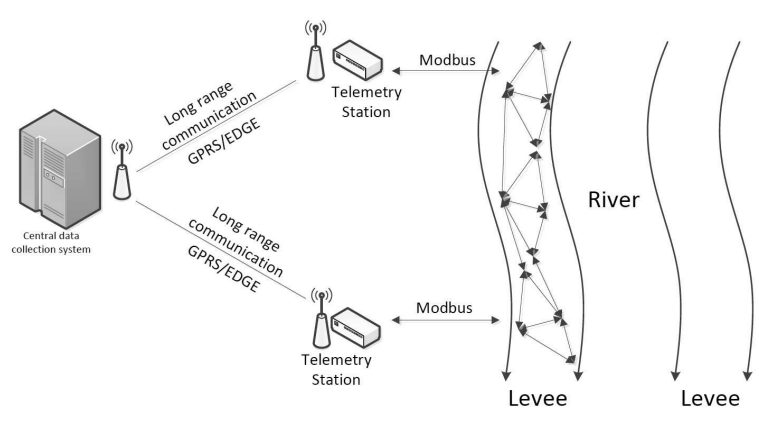

Fig. 1. Telemetry system for flood control levees

in Section V. Finally, the paper is summarized and further research steps are presented.

\section{MOTIVATING SCENARIO}

Recently, the importance of sensor network for monitoring various areas, objects or devices, has significantly increased. One of the areas in which telemetry and sensor network begin to fulfill a major role is monitoring systems for hydrologic engineering facilities in particular dams and flood levees[8], [9]. The overall concept of hydrological monitoring facilities was the starting point for the assumptions and implementation of the ISMOP research project which will result in guidelines for creating a telemetry system that enables continuous monitoring of levees. Research addresses the collection of massive measurement data in continuous mode, optimized transmission methodology, interpretation and analysis of monitored data with computer simulation and finally providing visualized results for the relevant authorities.

The biggest threat in the context of the levees is their leaking which could lead to their break. A typical application in this area is monitoring system of flood levees containing sensors that measure, among other things, temperature inside the levee. This measurement allows us to detect the rate of change of temperature in the levee. In the case of leakage when the water entering inside of the levee, water is the carrier of heat energy and increases or decreases the temperature in the levee. During the continuous measurement of temperature anomalies can be observed for a specific point in the levee, which deviate from the results of measurements made by the nearby sensors and observed the trend changes. This may indicate the presence of too much water in the levee and thus the danger of burst of the levee. The temperature and other physical values (such as pore pressure) allow to determine the condition of the levees and detect potential risks of leakage. An example scenario of telemetry system containing sensors placed inside the levees and the central data collection system is shown in Figure 1.

The role of the telemetry station is to acquire data from sensor networks which contain information about levee condition and to transmit these data to the central station. The data is generated by the sensor network for an epoch, which results in bursts of data each time the epoch changes. Apart from that, telemetry station also sends periodically information about its current condition e.g. battery level, CPU usage and others. The data from sensor networks, due to their importance, should be reliably delivered while the condition information may be transmitted on the best effort basis.

The use of telecommunications networks (including GPRS) for communication from/to telemetry stations is associated with specific energy consumption. It depends mostly on the time during which radio circuits are powered on and on the number of transmitted data. The motivation to our research is to reduce energy consumption during communication using GPRS from/to telemetry station.

\section{RELATED WORK}

Energy consumption of a wireless transmission device greatly depends on such factors as chosen communication standard, protocols used, and amount of transmitted data. Providing a medium-range or wide-area network connectivity requires a different approach. It is not a demanding task provided that a network infrastructure is available with appropriate SLA (Service Level Agreement) guaranties [10]. However, in remote areas a cellular connection is a common solution for industrial telemetry systems. Typical activities on a smartphone platform (sending a message, making a voice call, transmission over GPRS, etc.) are evaluated in [11]. An in-depth analysis o energy requirements for GPRS and UMTS services is provided in [12] and [13].

High-level protocols over cellular network also have an impact on overall energy requirements of a system [14]. A review of various middleware protocols for telemetry applications can be found in [15]. Message-oriented Middleware (MOM) is widely used as a communication layer for a variety of information systems which require event-driven message and data exchange, and more loose coupling than e.g. remote procedure calls. Examples of commonly utilized technologies for MOM are:

- Java Message Service (JMS) [16],

- Data Distribution Service [17],

- Extensible Messaging and Presence Protocol (XMPP) [18],

- MQ Telemetry Transport (MQTT) and its variation, MQTT-SN [19].

These technologies provide several other functionalities such as transaction management, broker clustering, additional message paradigms including point-to-point, publish/subscribe and request-response. Nevertheless, only MQTT has been designed especially for transferring telemetry-style binary data from the pervasive devices with limited computational resources. It should be noted that utilizing the MQTT protocol over a standard TCP connection may provide redundant message delivery guaranties. As TCP is intended to provide a reliable link and has built-in retransmission mechanisms, setting the MQTT's QoS parameter to 1 or 2 provides another (redundant) layer of persistence. In contrast, those higher levels of QoS seem very useful in MQTT-SN variation which by design uses UDP datagrams. In our research we have chosen MQTTSN messaging protocol (formerly MQTT-S [20]) because it is promising due to its simplicity. MQTT-SN clients can 


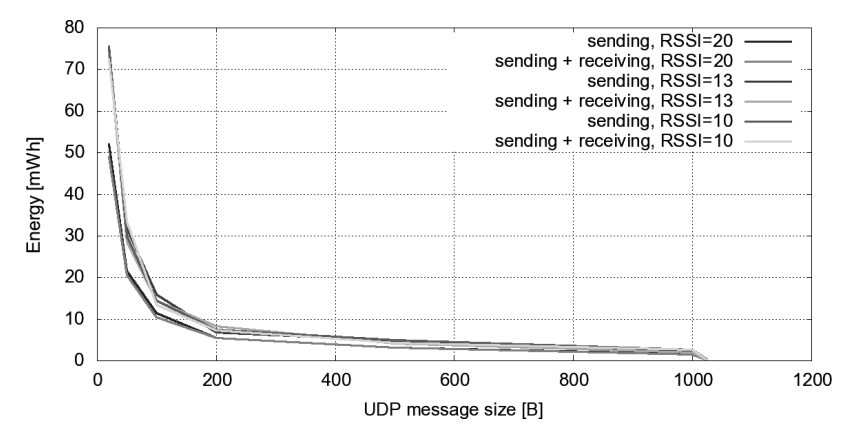

Fig. 2. Power necessary to send $10 \mathrm{kB}$ of data using GPRS communication as a function of packet size

be implemented in resource-constrained hardware (embedded systems), and there are available plenty of its implementations.

However, it is difficult to find any power efficiency considerations for MQTT-SN. By far many solutions dedicated to the MOM technology have been optimized to limit the data transfer and save energy. The MQTT-SN is an example of protocol that was optimized in terms of quantity of data to be transmitted. It results from using short-distance wireless protocols for sensor-based data transmission, including the IEEE 802.15.4 protocol. All these issues, not previously mentioned in MOM solutions, and particularly in the MQTT-SN protocol, have been analyzed in this article and relevant solutions have been suggested.

\section{AdAPtiVE MESSAGE AGGREGATION}

The main goal of the research was to decrease the amount of energy necessary to send the data using MOM over GPRS connectivity. The results of the base research aimed to analyze how much energy is used by GPRS modem as a function of packet data size is depicted in Figure 2.

The nonlinearity in the power consumption is caused by two factors: overhead of the appropriate headers of OSI/ISO stack and purely technical considerations related to the physical communication with the GPRS modem in embedded devices (e.g. the time of data preparation, inter frame gaps and others). Figure 3 shows the overall data size that are necessary to send $10 \mathrm{~KB}$ of data payload. The overhead is related to the headers of UDP and IP protocols for each packet. Consequently, from an energy consumption point of view, it is much better for fixed amount of data to send it using as large packet size as possible.

On the other hand, the amount of measurement data that need to be transmitted is usually small i.e. typically $20 \mathrm{~B}$. The previous measurements show that sending such small packets would be inefficient. Based on these data, we propose the concept of data aggregation before transmission.

Our concept, as presented in Figure 4, can be applied to MQTT messages with QoS 0 and 1. In QoS 0, messages are not acknowledged, so client may aggregate several messages before sending them. In the second case, with QoS 1, all the messages had to be acknowledged so, we propose also aggregating the acknowledgment packets on the broker side.

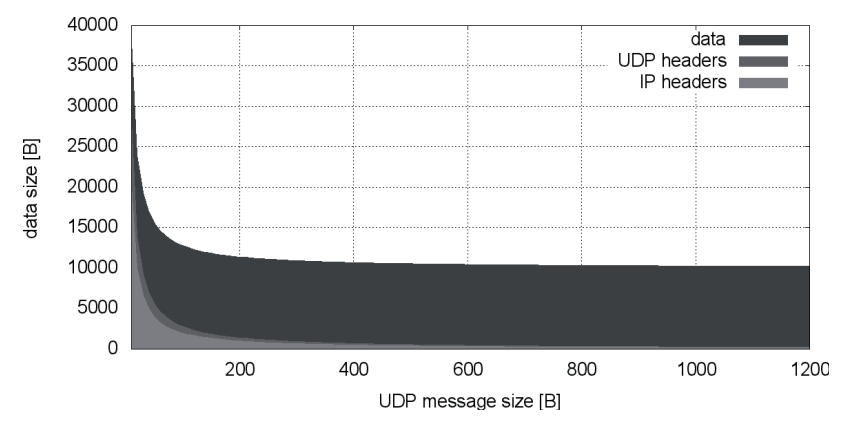

Fig. 3. Overall data necessary to send $10 \mathrm{kB}$ of data as a function of packet size

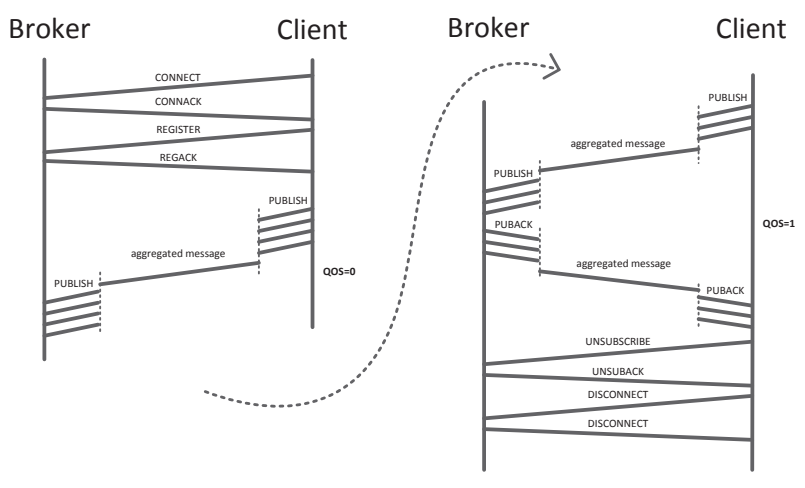

Fig. 4. Message aggregation concept for MQTT-SN

We have assumed that larger, important data to send appear in the bursts, while less important data are sent on regular basis in small chunks. This is dictated by the fact that underlying sensor network (installed in the levee) wakes up in time intervals to preserve power. The naive approach to data aggregation is presented in Figure 5. The main idea is that new messages are not send immediately but first copied to the buffer $B$ with fixed length $L$ and then sent as an aggregated packet. There are two conditions that decide of sending data: buffer is overflowed or buffer timeout $T^{I}$ has ended. During the period 1 and 3 aggregated messages are sent because of the timeout condition, while during the period 2 aggregated message is sent because of the overflow condition. The drawback of the method is that in the period 3, the messages that belong to the burst are sent with longer delay then previous ones because overflow condition did not occurred. Such a situation is unwanted if the data has to be analyzed in the real-time.

In our method, we propose adaptive timeout calculation that adjust itself to the frequency of incoming data. The main concept is that buffer overflow situation decreases the buffer timeout meaning that messages should be send faster, while decreasing the frequency of incoming new data recovers the timeout to its previous value. Such a policy results in the situation that messages belonging to the data burst are received by the broker in the burst as well. The concept is depicted in 


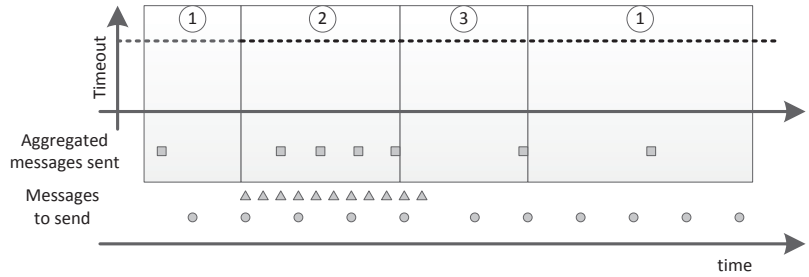

Fig. 5. Naive approach to data aggregation (time periods are marked with numbers, telemetry data are represented by small grey circles, triangles represent bursts of measurement data, packed and transmitted data are depicted as squares)

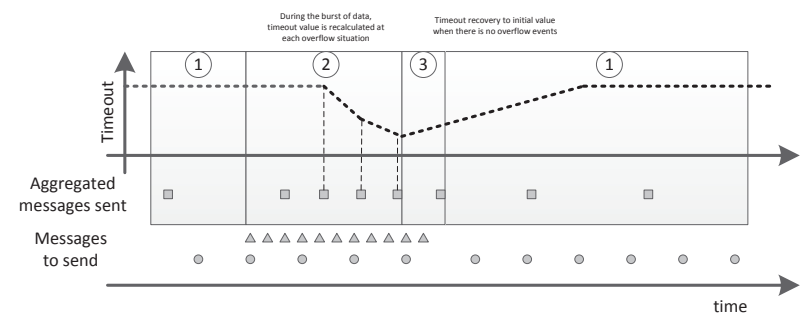

Fig. 6. Adaptive approach to data aggregation (time periods are marked with numbers, telemetry data are represented by small grey circles, triangles represent bursts of measurement data, packed and transmitted data are depicted as squares)

Figure 6. During the period 3, the aggregated message is send earlier than in naive approach to the aggregation.

As can be noticed, the presented adaptive message aggregation does not generate any additional overhead. On the contrary, it contributes to reducing the overhead introduced by the OSI/ISO network communication stack when transmitting small chunks of data. Another aspect is the latency of data arrival. It results directly from the fact, that data needs to be packed in a buffer. In our solution, the latency is controlled with the variable timeout value. The timeout automatically decreases as more data arrives. This can be utilized to keep latency and overhead ratio at reasonable levels.

More formally, the algorithm is composed of the two parts and might be presented as follows. The input to the algorithm is provided by four values: $T^{I}$ is the initial timeout value, $T^{R}$ is the recovery time to the initial value, the factor $\alpha$, and a buffer length $L$. In the MOM client there is global timer $T$ that represents actual timeout value - at time $t$ this value is denoted as $T_{t}$. When the aggregated message buffer $B$ of length $L$ is created at time $t$, it has assigned timeout that equals $T_{t}$. Length of the buffers is constant.

First part of the algorithm is responsible for recovering (i.e. increasing) timeout $T$ to the initial value of $T^{I}$ and is formulated as follow: for each time $k$, the timeout $T_{k+1}$ is calculated using the equation 1 , where $\Delta T$ is the time step.

$$
T_{k+1}=\min \left(T_{k}+\frac{T^{I}}{T^{R}} \Delta T, T^{I}\right)
$$

The second part of the algorithm is responsible for decreasing timeout $T$ to the value that is similar to the time of sending overflowed buffers when data burst is observed. The equation 2 is used only when the overflow of the buffer is observed. Value $d$ in the equation is the time from the last overflow event.

$$
T_{k+1}=\min \left(\alpha T_{k}+(1-\alpha) d, T^{I}\right)
$$

Having in mind, that data usually comes from remote telemetry stations to the central point, we propose to use adaptive aggregation method on the client side to aggregate messages, and to use naive aggregation approach on the broker side to aggregate acknowledgments. The evaluation results of the proposed algorithm are presented in the next section.

\section{Evaluation}

We have evaluated the proposed concept on the scenario similar to the one presented in the previous section. We have assumed that data from levee monitoring sensors are gathered and sent in two stages:

- at the beginning, for QoS 1 in MQTT/MQTT-SN, 1000 PUBLISH messages with a length of $20 \mathrm{~B}$ are sent and received confirmation of these messages (PUBACK),

- later, for QoS 0 in MQTT/MQTT-SN, in 12 minutes epoch and for every $30 \mathrm{~s}$ PUBLISH messages with a length of $20 \mathrm{~B}$ are transmitted.

During tests we used a popular GPRS modem (SIM900D) and, in order to verify the results obtained, we also used an industrial GPRS Modem (Wavecom Fastrack Supreme 20). In order to develop test software we extend implementation of MQTT-SN - Eclipse Mosquitto [21] (which we call A-MQTT$S N)$ to support adaptation.

Above presented testing scenario was carried out for three cases using:

- MQTT protocol (Eclipse Mosquitto),

- MQTT-SN protocol (Eclipse Mosquitto),

- A-MQTT-SN protocol with adaptation for sent and received data (message type PUBLISH and PUBACK).

The adaptive aggregation algorithm for A-MQTT-SN was initiated with values: $T I=120 s, T R=240 s, \alpha=0.5$, and $L=1000 \mathrm{~B}$. The naive aggregation algorithm was initiated with values: $T_{I}=2 \mathrm{~s}$ and $L=250 \mathrm{~B}$. The values are application-specific, and should be tailored for different conditions, such as: amount of transmitted data, realtime boundaries and the maximal accepted latency by the application.

The measurements were made with a custom multichannel current and voltage sensing module and tailored for energy measurements of various embedded devices. Data for all of the presented tests was acquired from the GPRS modems (Class 10) connecting to public GSM network with a throughput of $25 \mathrm{~Kb} / \mathrm{s}$ (2 timeslots in uplink direction).

The result of these tests is shown in the following figures:

- for MQTT protocol (using TCP and PPP protocols) Figure 7,

- for MQTT-SN protocol (using UDP, PPP protocols and AT commands on the GPRS modem) - Figure 8, 

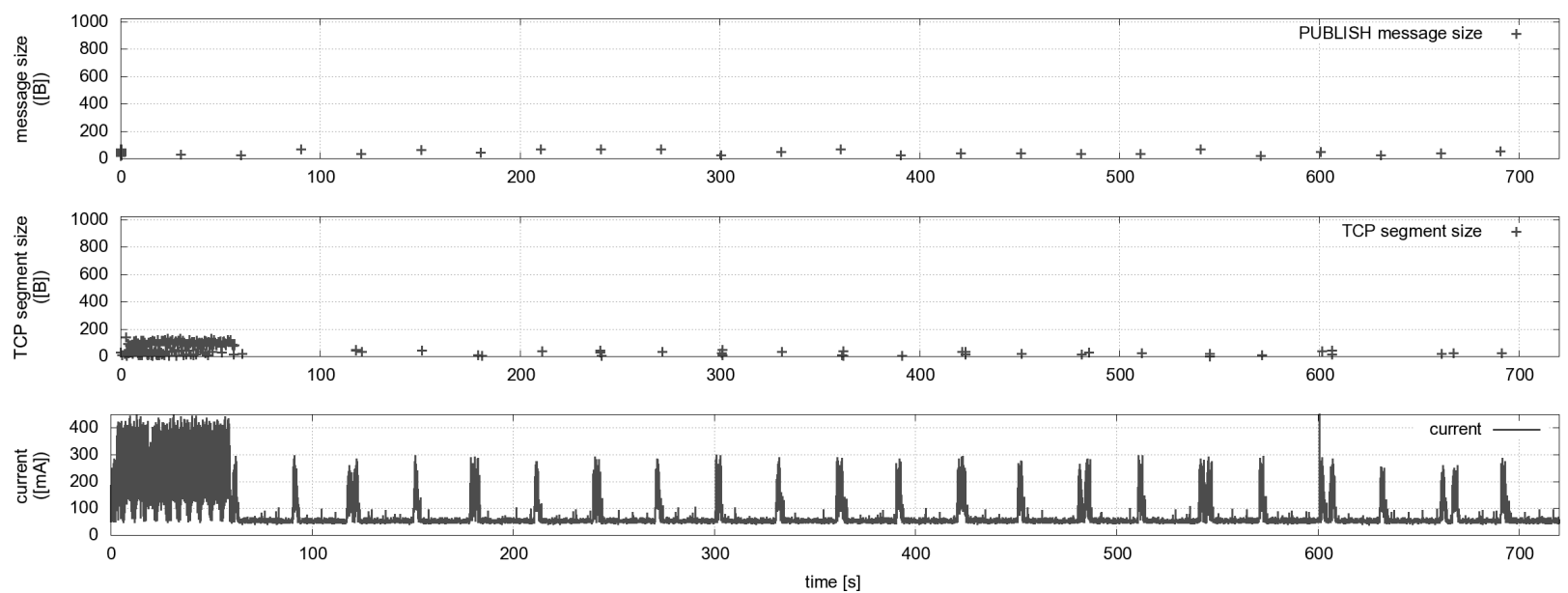

Fig. 7. The current consumption for the MQTT protocol
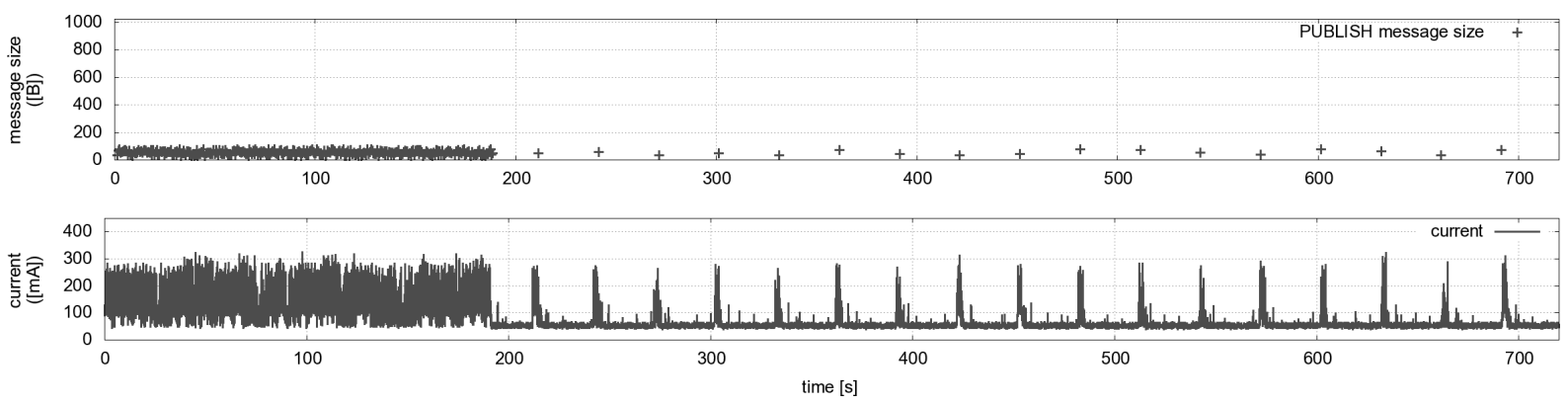

Fig. 8. The current consumption for the MQTT-SN protocol
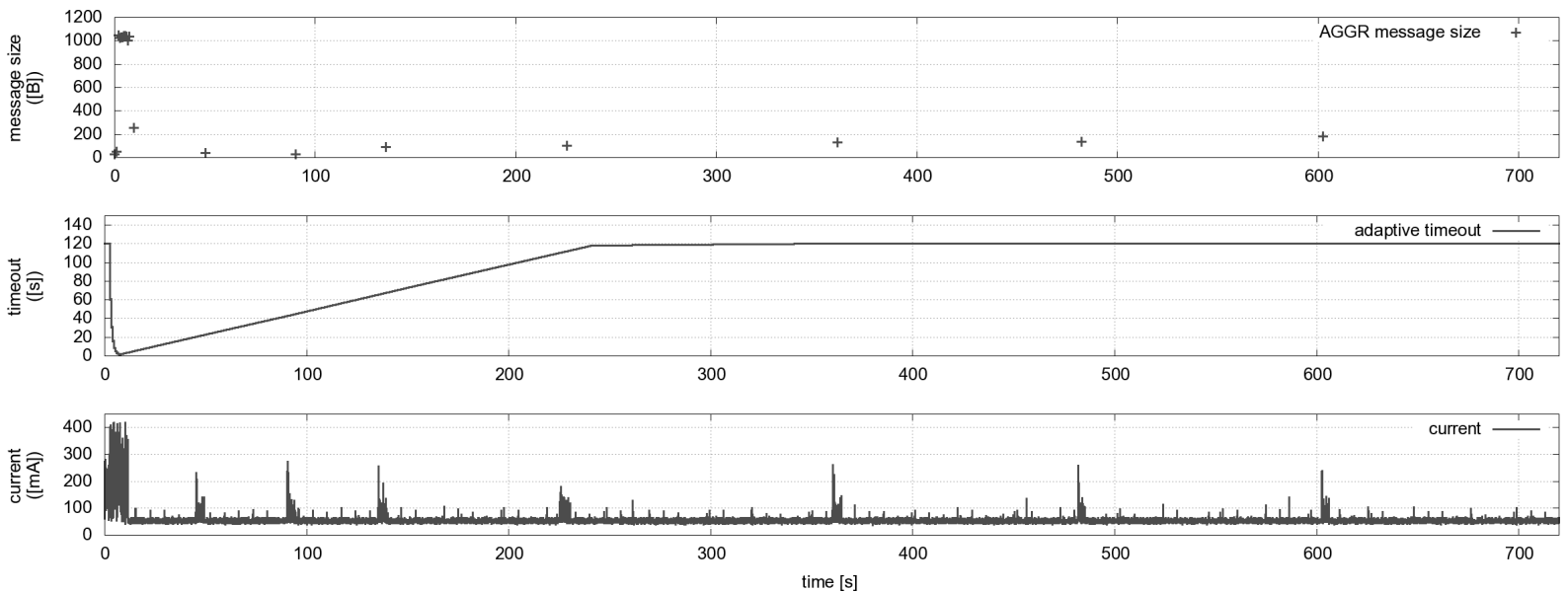

Fig. 9. The power consumption for the A-MQTT-SN protocol

- for A-MQTT-SN protocol (using UDP, PPP protocols and AT commands on the GPRS modem) - Figure 9.

Analyzing the results we can observe that the power consumption of a GPRS modem for data transmission is higher for MQTT and MQTT-SN than A-MQTT-SN protocol. In our opinion higher power consumption for MQTT protocol is the result of using TCP and its complexity (call setup, retransmissions). It can be seen that in the case of sending 


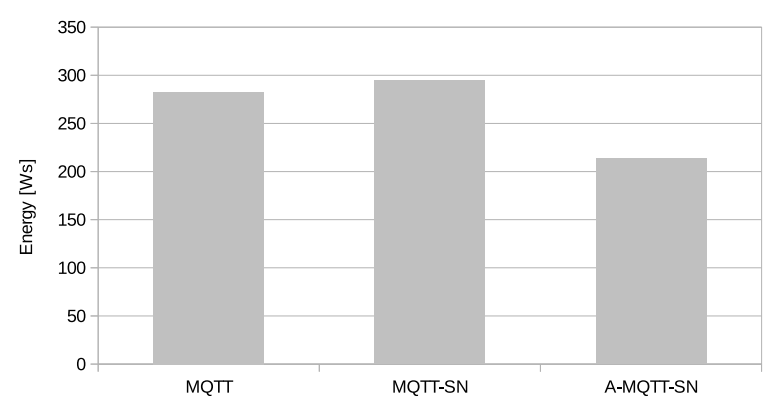

Fig. 10. Energy consumption for MQTT, MQTT-SN and A-MQTT-SN protocols

MQTT messages with QoS 0 should not be a retransmission of messages which, however, are made because of the use of TCP protocol. In the second case, where we used UDP and MQTT-SN protocols, increased energy consumption via modem is related to the size of transmitted data. In case of sending a large number of small packets the overhead associated with packet header is visible. The developed AMQTT-SN protocol variation aggregates data transmitted and significantly decreases the number of headers and thus gives the best result as regards the energy consumption during transmission by GPRS modem (Figure 10).

\section{Summary AND Future Work}

The paper discusses the problem of sending the data using GPRS connectivity from remote telemetry stations. We have analyzed various communication protocols and finally selected MQTT-SN. The paper proposed the extensions to the communication protocol that adjust its behavior to the GPRS connectivity profile in order to decrease the data transmissionrelated energy consumption.

The motivating scenario presented in the paper is only one of the possible applications of our concept. The solutions might be successfully applied to e.g. multilayer telemetry solutions where due to the sleepy nodes, data have to be pushed rarely but efficiently.

\section{ACKNOWLEDGMENT}

The research presented in this paper was partially supported by the National Centre for Research and Development (NCBiR) under Grant No. PBS1/B9/18/2013 and by the Polish Ministry of Science and Higher Education under AGH University of Science and Technology Grant 11.11.230.015 (statutory project).

\section{REFERENCES}

[1] M. Szmechta and P. Aksamit, "Modeling packet delay distributions in an industrial telemetry system," in Computational Intelligence and Intelligent Informatics (ISCIII), 2011 5th International Symposium on. IEEE, 2011, pp. 71-74.
[2] T. Szydlo, S. Gut, and B. Puto, "Smart Applications: Discovering and interacting with constrained resources IPv6 enabled devices," Przeglad Elektrotechniczny, pp. 221-226, 062013.

[3] T. Szydlo, P. Suder, and J. Bibro, "Message Oriented Communication For IPV6 Enabled Pervasive Devices," Computer Science, vol. 14, no. 4, 2013.

[4] E. Curry, "Message-Oriented Middleware," in Middleware for Communications, Q. H. Mahmoud, Ed. Chichester, England: John Wiley and Sons, 2004, ch. 1, pp. 1-28.

[5] "ISMOP Project," www.ismop.edu.pl, 2013, accessed: 2014-04-19.

[6] A. Piórkowski and A. Leśniak, "Using data stream management systems in the design of monitoring system for flood embankments," Studia Informatica, vol. 35, no. 2, pp. 297-310, 2014.

[7] M. Chuchro, M. Lupa, A. Pięta, A. Piórkowski, and A. Leśniak, "A concept of time windows length selection in stream databases in the context of sensor networks monitoring," in New Trends in Databases and Information Systems, Proceedings of 18th East-European Conference on Advances in Databases and Information Systems (in print), ser. Advances in Intelligent Systems and Computing. Springer, 2015.

[8] B. Balis, T. Bartynski, M. Bubak, G. Dyk, T. Gubala, and M. Kasztelnik, "A development and execution environment for early warning systems for natural disasters," in Cluster, Cloud and Grid Computing (CCGrid), 2013 13th IEEE/ACM International Symposium on, May 2013, pp. 575582.

[9] B. Balis, M. Kasztelnik, M. Bubak, T. Bartynski, T. Gubaa, P. Nowakowski, and J. Broekhuijsen, "The urbanflood common information space for early warning systems," Procedia Computer Science, vol. 4, no. 0, pp. 96 - 105, 2011, proceedings of the International Conference on Computational Science, $\{$ ICCS 2011.

[10] J. Kosinski, P. Nawrocki, D. Radziszowski, K. Zielinski, S. Zielinski, G. Przybylski, and P. Wnek, "SLA Monitoring and Management Framework for Telecommunication Services," in Networking and Services, 2008. ICNS 2008. Fourth International Conference on, J. Bi, K. Chin, C. Dini, L. Lehmann, and D. C. Pheanis, Eds. IEEE Computer Society, 2008, pp. 170-175

[11] G. P. Perrucci, F. H. Fitzek, and J. Widmer, "Survey on energy consumption entities on the smartphone platform," in Vehicular Technology Conference (VTC Spring), 2011 IEEE 73rd. IEEE, 2011, pp. 1-6.

[12] A. Sikora, A. Yunitasari, and M. Dold, "GPRS and UMTS services for ultra low energy M2M-communication," in Intelligent Data Acquisition and Advanced Computing Systems (IDAACS), 2013 IEEE 7th International Conference on, vol. 1. IEEE, 2013, pp. 494-498.

[13] F. Pauls, S. Krone, W. Nitzold, G. Fettweis, and C. Flores, "Evaluation of Efficient Modes of Operation of GSM/GPRS Modules for M2M Communications," in Vehicular Technology Conference (VTC Fall), 2013 IEEE 78th. IEEE, 2013, pp. 1-6.

[14] E. J. Vergara Alonso, "Exploiting Energy Awareness in Mobile Communication," 2013.

[15] A. Azzara, S. Bocchino, P. Pagano, G. Pellerano, and M. Petracca, "Middleware solutions in WSN: The IoT oriented approach in the ICSI project," in Software, Telecommunications and Computer Networks (SoftCOM), 2013 21st International Conference on. IEEE, 2013, pp. $1-6$.

[16] "Java Message Service documentation," http://docs.oracle.com/javaee/5/tutorial/doc/bncdq.html, accessed: 2014-04-19.

[17] "Data Distribution Service ver. 1.2 documentation," http://www.omg.org/spec/DDS/1.2, accessed: 2014-04-19.

[18] "Extensible Messaging and Presence Protocol documentation," http://xmpp.org/xsf/press/2004-10-04.shtml, accessed: 2014-04-19.

[19] "MQ Telemetry Transport (MQTT) documentation," http://mqtt.org/documentation, accessed: 2014-04-19.

[20] U. Hunkeler, H. L. Truong, and A. Stanford-Clark, "MQTT-SA publish/subscribe protocol for Wireless Sensor Networks," in Communication Systems Software and Middleware and Workshops, 2008. COMSWARE 2008. 3rd International Conference on. IEEE, 2008, pp. 791798.

[21] "Mosquitto technology project," http://projects.eclipse.org/projects/ technology.mosquitto, accessed: 2014-04-19. 